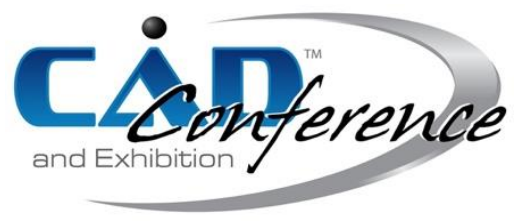

Title:

\title{
Data Augmentation of Classifiers for Components in Industrial Plants Using CAD Models
}

Authors:

Kohei Shigeta, s2032054@edu.cc.uec.ac.jp, The University of Electro-Communications

Daiki Hanai, h1710495@edu.cc.uec.ac.jp, The University of Electro-Communications

Hiroshi Masuda, h.masuda@uec.ac.jp, The University of Electro-Communications

Keywords:

Point Cloud, Convolutional Neural Network, Terrestrial Laser Scanner, Poiny Processing, Reverse Engineering, Shape Reconstruction

DOI: 10.14733/cadconfP.2021.288-292

Introduction:

3D models of industrial plants are very useful for simulating maintenance work. However, in the case of facilities built in the old age and repeatedly renovated, 3D models often do not exist, and even reliable drawings may not exist. This problem can be solved by creating 3D models from point clouds captured using a terrestrial laser scanner (TLS).

Since large industrial plants contain a large number of components, it is necessary to identify and extract each component from point clouds. This task is not easy because it is difficult to obtain a complete point cloud of each component due to limited scanning positions. In industrial plants, the shape of many components is determined by the industrial standards, and most components are composed of simple surfaces such as cylinders and planes. Several methods have been proposed to identify standard components using connection rules between components [1], [3]. However as shown in Fig 1, some components have shapes that are not defined by the industrial standards, and it is difficult to uniquely identify such components using these methods.

In recent years, machine learning has become a common tool, and especially convolutional neural network $(\mathrm{CNN})$ has achieved excellent scores for images. If point clouds of components can be adequately encoded as feature vectors, it may be possible to identify them from point clouds. However, $\mathrm{CNN}$ requires a large number of training data with labels to achieve a high classification capability. It is actually very costly and time-consuming to acquire point clouds of many industrial plants and segment the point clouds into each component with a label.

To remedy this problem, it is desirable to train CNN using CAD data instead of captured point clouds. In this paper, we discuss methods for augmenting training data by using virtual point clouds generated from CAD models.

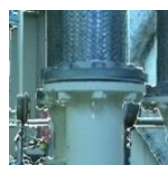

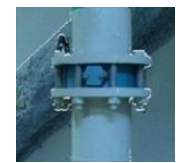

(a)
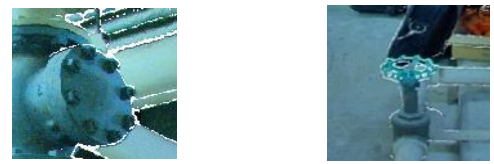

(b)

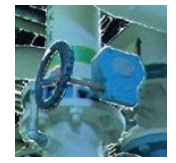

Fig. 1: Components with various shapes: (a) Flange and (b) Valve.

Generation of 2D Images from Point Clouds:

In our previous work [4], we proposed a method for identifying components in industrial plants by applying CNN to RGB, intensity, and depth images that were generated from point clouds captured using a TLS. Fig. 2 shows a process for generating a 2D image from a point cloud. Since coordinates acquired 
by a TLS are calculated from the distance $d$, the azimuth angle $\theta$, and zenith angle $\phi$ of laser beams, a point cloud can be mapped on the $(\theta, \phi)$ plane, as show in Fig. 2(a), (b). Since the image defined by $\theta$ and $\phi$ is distorted, the coordinate $(\theta, \phi)$ is converted to the coordinate $(I, J)$ on the perspective projection plane, as shown in Fig. 2(c). The converted image preserves the linearity, as shown in Fig. 2(e).

Generation of Depth and Intensity Images from CAD Models:

To train a CNN classifier, a large number of images are required. Therefore, we generate 2D images from CAD models. Fig. 3 shows a process for 2D image generation. First, dense points are randomly generated on faces of a CAD model. For generating points, a triangle face is randomly selected with a probability proportional to the area. Then, point $\boldsymbol{P}$ is generated on the face using the vertices $\boldsymbol{A}, \boldsymbol{B}, \boldsymbol{C}$ of the triangle and two random values $\alpha$ and $\beta$ within $[0,1]$, as:

$$
\boldsymbol{P}=(1-\sqrt{\alpha}) \boldsymbol{A}+\sqrt{\alpha}(1-\beta) \boldsymbol{B}+\sqrt{\alpha} \beta \boldsymbol{C}
$$

Fig. 3(b) shows points generated on the CAD model. The generated points are randomly rotated around the $\mathrm{X}, \mathrm{Y}$, and $\mathrm{Z}$ axes, and moved to random positions. Then, each point is projected on the image plane, as shown in Fig. 3(c). On each pixel of the 2D image, the depth or intensity value is described. By iterating this process many times, a large number of training data can be created from a CAD model. Fig. 3(d) shows depth images generated from a CAD model.

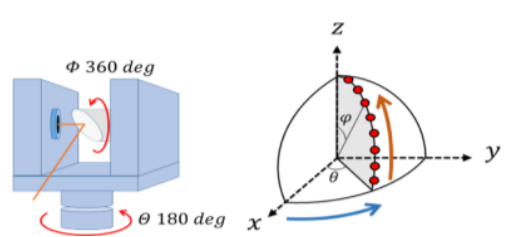

(a)

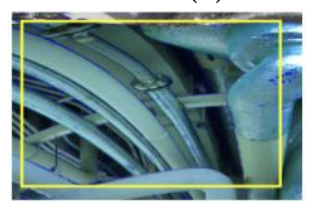

(c)

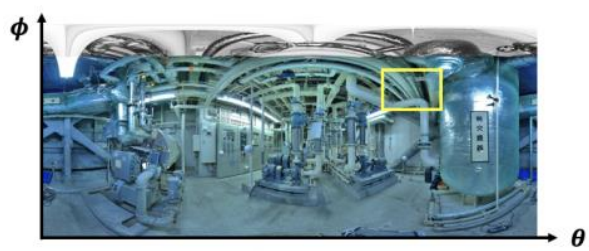

(b)

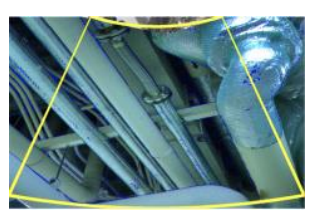

(e)

Fig. 2: Generation of 2D images from point clouds: a) Angles of laser beam, (b) RGB image on the $(\theta, \phi)$ plane. (c) Close-up, (d) Perspective projection, and (e) Perspective image.

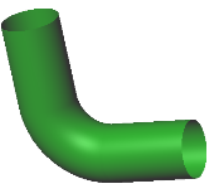

(a)

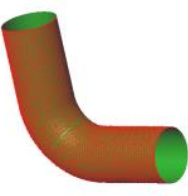

(b)
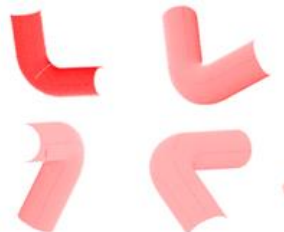

(c)
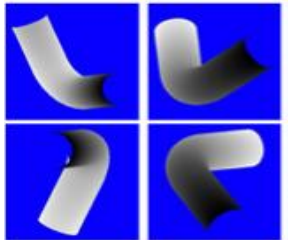

(d)

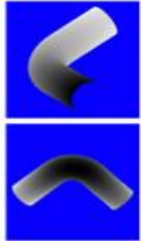

CAD model,

Fig. 3: Images generated from CAD model: (a) CAD model,
(c) Points projected on the image plane, and (d) Depth images.

Estimation of Intensity of CAD Models:

Typical TLSs output an intensity value for each point. The intensity value is calculated from the strength of the laser beam reflected from an object. The strength of the returned laser beam depends on the distance from the scanner, the irradiation angle, the material and color of the object, and so on. When the distance from the scanner is larger, the strength of the reflected light becomes smaller. The intensity values are calculated by compensating for the effect of distances so that the intensity image looks like a natural black and white image. 
To generate intensity images from CAD models, intensity values have to be estimated. However, the intensity value is calculated using a scanner-specific algorithm, and the vendor proprietary module is required for calculating intensity values from raw data. Therefore, we estimate the intensity values of virtual point clouds using actual point clouds. We suppose that the intensity values depend only on irradiation angles. First, irradiation angles and intensity values are calculated from actual point clouds. To obtain irradiation angles, the normal vector at each point has to be calculated. Although the normal vector at each point can be calculated by fitting a plane to neighbor points, it is noisy and unstable. Therefore, we fit a cylinder to points of each cylindrical component using the RANSAC method [2]. The normal vector at each measured point is obtained as the normal at the nearest point on the cylindrical surface. The irradiation angle $\theta$ at the point is calculated as the angle between the normal vector and the laser beam.

In industrial plants, there are a lot of cylindrical components. Since a cylindrical surface contains a wide range of normal vectors, intensity values for various irradiation angles can be obtained. To estimate the intensity value, we approximate the intensity value as $I=f(\cos \theta)$ using a polynomial function $f$. In this study, the piecewise polynomial function is calculated simply by fitting a cubic B-spline curve.

Intensity images can be generated from CAD models using estimated intensity values. Fig. 4 shows samples of intensity images generated from CAD models.
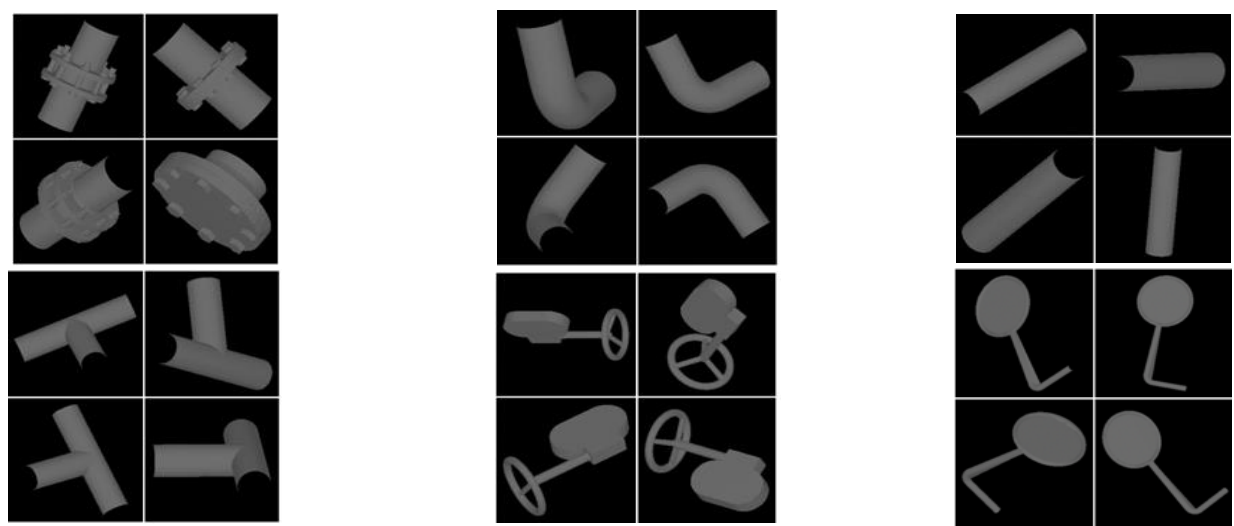

Fig. 4: Intensity images created from CAD models.

Augmentation of Point Clouds Generated from CAD Model:

In CNN, datasets are typically augmented to avoid overfitting to specific data. The number of training images is often increased by rotating, flipping, scaling, shearing, and adding noises to the original images. In addition to these augmentations, images created from point clouds should reflect the characteristics of point cloud measurement. In general, missing portions are generated due to occlusions and outliers in actual point clouds. In this sense, virtual point clouds generated from CAD models are unrealistically clean compared to actual point clouds. In this research, we focus on augmentation by adding missing portions to point clouds generated from CAD models.

In this research, missing portions are approximated as circular regions or polygonal regions. For generating a circular missing portion, a point is randomly selected and the $k$-nearest neighbor points are removed from the point cloud. This process are repeated $n$ times to eliminate $n$ circular regions. Then, the depth image is generated using the remaining points.

For removing a polygonal region, a pixel position is randomly selected on the depth image. Then, $m$ points are randomly selected from a range within the distance $d$ from the selected pixel. An $m$-sided polygon is generated by connecting the selected $m$ points, and the polygonal region is removed from the depth image. This process are repeated $n$ times to eliminate $n$ polygonal regions.

Fig. 5 shows a process of point removal. In the upper case, circular regions are removed from the depth image. In the bottom case, polygonal regions are removed. 
Experimental Results:

In order to evaluate our method, we trained classifiers and verified the accuracy. It is considered that point clouds generated from CAD models are effective when the number of actual point clouds is small.

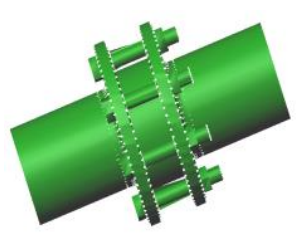

(a)

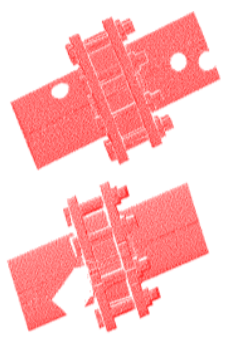

(b)

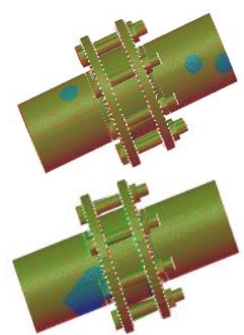

(c)

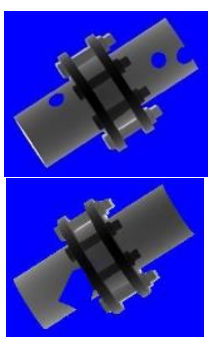

(d)

Fig. 5: Augmenting virtual point clouds (Upper: Removing circular regions, Bottom: Removing polygonal regions): (a) CAD model, (b) Partly missing point cloud, (c) Missing portions shown in blue, and (d) Incomplete depth image.

Therefore, we used 2 sets of actual point clouds with different numbers of components as training data. Tab. 1 shows the numbers of actual point clouds, virtual point clouds, and test data.

We trained the classifiers by transfer learning based on the pre-trained VGG16 [4]. For evaluating the effect of virtual intensity images, we created two types of classifiers. One used only intensity images generated from actual point clouds, and the other used intensity images generated both actual and virtual point clouds. The results are shown in Tab. 2. The results show that the accuracy was improved by using virtual intensity images. In particular, the F-measures has improved considerably for components with a very small number of training data, such as Tees.

Then we evaluated classifiers using depth images. We created four classifiers using depth images generated from (1) only actual point clouds, (2) both actual and virtual point clouds without augmentation, (3) actual point clouds and virtual point clouds augmented by removing circle regions, and (4) actual point clouds and virtual point clouds augmented by removing polygonal regions. The results are shown in Tab. 3. The accuracy was improved by augmented point clouds. In Case 1, Fmeasures for circle region removal and polygonal region removal were almost the same. It is considered that missing portions were effective to enhance a generalization capability. Tab. 4 shows F-measures for Case 2 using classifiers for (3) and (4). The result shows that the classifiers trained using augmented depth images achieved good F-measures even when the number of actual point clouds was small. In these evaluations, F-measures were better when circle regions were removed.

\section{Conclusion:}

In this research, we proposed a method for generating intensity and depth images from CAD models. Intensity values of virtual point clouds were estimated from actual point clouds of cylindrical objects. In addition, we described a method for augmenting virtual point clouds by generating missing portions. In our evaluation, these methods were effective to improve the accuracy of classification.

In future work, we would like to investigate other methods for augmenting virtual point clouds. In addition, we would like to investigate methods for generating incomplete point clouds based on CNN. Moreover, we would like to consider modeling components using the created classifier.

\begin{tabular}{c|cc|c|c}
\hline & \multicolumn{3}{|c|}{ Training data } & \multirow{2}{*}{ Test Data } \\
\cline { 2 - 3 } & \multicolumn{2}{|c|}{ Actual Point Clouds } & Virtual Point Clouds & \\
\cline { 2 - 3 } & Case1 & Case2 & for Augmentation & 80 \\
Flange & 80 & 40 & 120 & 85 \\
Elbow & 86 & 43 & 80 & 40 \\
Straight & 40 & 20 & 80 & 10 \\
Tee & 11 & 6 & 80 & 20 \\
Valve & 22 & 11 & 80 &
\end{tabular}




\begin{tabular}{|c|c|c|c|c|}
\hline Manometer & 4 & 2 & 80 & 3 \\
\hline total & 243 & 122 & 520 & 238 \\
\hline \multicolumn{5}{|c|}{ Tab. 1: Numbers of training data. } \\
\hline & \multicolumn{2}{|c|}{ Case1 } & \multicolumn{2}{|c|}{ Case2 } \\
\hline & Actual Intensity & Actual \& Virtual & Actual Intensity & Actual \& Virtual \\
\hline Flange & $91.7 \%$ & $91.3 \%$ & $89.3 \%$ & $90.1 \%$ \\
\hline Elbow & $90.1 \%$ & $91.7 \%$ & $89.3 \%$ & $90.6 \%$ \\
\hline Straight & $95.1 \%$ & $97.5 \%$ & $91.6 \%$ & $94.7 \%$ \\
\hline Tee & $73.7 \%$ & $85.7 \%$ & $42.9 \%$ & $58.8 \%$ \\
\hline Valve & $81.0 \%$ & $82.9 \%$ & $77.8 \%$ & $81.1 \%$ \\
\hline Manometer & $80.0 \%$ & $80.0 \%$ & $85.7 \%$ & $80.0 \%$ \\
\hline Average & $89.9 \%$ & $91.4 \%$ & $86.7 \%$ & $88.9 \%$ \\
\hline
\end{tabular}

Tab. 2: F-measures of classifiers using intensity images.

\begin{tabular}{c|cccc}
\hline & Only Actual & Actual \& Virtual & $\begin{array}{c}\text { Actual \& Virtual } \\
\text { (Circle Removal) }\end{array}$ & $\begin{array}{c}\text { Actual \& Virtual } \\
\text { (Polygon Removal) }\end{array}$ \\
\hline Flange & $94.3 \%$ & $97.4 \%$ & $98.1 \%$ & $98.1 \%$ \\
Elbow & $92.1 \%$ & $95.5 \%$ & $96.0 \%$ & $95.3 \%$ \\
Straight & $92.5 \%$ & $97.5 \%$ & $96.3 \%$ & $97.5 \%$ \\
Tee & $62.5 \%$ & $75.0 \%$ & $84.2 \%$ & $85.7 \%$ \\
Valve & $97.4 \%$ & $95.2 \%$ & $100.0 \%$ & $100.0 \%$ \\
Manometer & $100.0 \%$ & $100.0 \%$ & $100.0 \%$ & $100.0 \%$ \\
\hline Average & $92.2 \%$ & $95.6 \%$ & $96.6 \%$ & $96.7 \%$ \\
\hline
\end{tabular}

Tab. 3: F-measures of classifiers using depth images (for Case 1).

\begin{tabular}{c|cc}
\hline & Circle Removal & Polygon Removal \\
\hline Flange & $96.2 \%$ & $95.6 \%$ \\
Elbow & $95.4 \%$ & $90.8 \%$ \\
Straight & $96.1 \%$ & $85.7 \%$ \\
Tee & $85.7 \%$ & $77.8 \%$ \\
Valve & $90.0 \%$ & $94.7 \%$ \\
Manometer & $100.0 \%$ & $100.0 \%$ \\
\hline Average & $95.0 \%$ & $91.5 \%$ \\
\hline
\end{tabular}

Tab. 4: F-measures of classifiers using actual and virtual point clouds (for Case 2).

References:

[1] Kawashima, K.; Kanai, S.; Date, H.: As-built modeling of piping system from terrestrial laser-scanned point clouds using normal-based region growing, Journal of Computational Design and Engineering, 1(1), 2014, 13-26. https://doi.org/10.7315/JCDE.2014.002

[2] Masduda, H.; Niwa, T.; Tanaka, I.; Matsuoka, R.: Reconstruction of polygonal faces from large-scale point-clouds of engineering plants, Computer-Aided Design and Applications, 12(5), 2014, 150-152. https;//dx.doi.org/10.14733/cadconfp.2014.150-152

[3] Matsuoka, R.; Masuda, H.: Reconstruction of Structure Shapes of Facilities from Large-scale point cloud (1st report) - Estimation and evaluation of connecting components based on extracted surfaces, Journal of the Japan Society of Precision, 2014, 604-608. 10.2493/jjspe.80.604

[4] Shigeta, K; Masuda, H: Extraction and Recognition of Components from Point Clouds of Industrial Plants, Computer-Aided Design \& Applications, Vol. 18, No. 5, 2021, 890-899. https://doi.org/10.14733/cadaps.2021.890-899

[5] Simonyan, K.; Zisserman, A.: Very Deep Convolutional Networks for Large-Scale Image Recognition, Eighth International Conference on Learning Representations, 2015.arXiv:1409.1556 